\title{
Ortho-Positronium in Some $n$-Alkanes; Influence of Temperature and Pressure
}

\author{
B. Zgardzińska, M. Pietrow, T. GoworeK* \\ AND J. WAWRYSZCZUK \\ Department of Nuclear Methods, Institute of Physics, Maria Curie-Skłodowska \\ University, pl. Marii Curie Skłodowskiej 1, 20-031 Lublin, Poland \\ Positron lifetime spectra were measured for a series of odd-numbered \\ $n$-alkanes from $\mathrm{C}_{11} \mathrm{H}_{24}$ to $\mathrm{C}_{19} \mathrm{H}_{40}$ as a function of temperature and pressure. \\ The ortho-Ps lifetimes in the rotator phase and the increase in intensity at \\ the transition to that phase can be explained by location of Ps in the vicinity \\ of kink-type conformers. The relation between o-Ps lifetime and molecule \\ length can be described in the framework of extended Tao-Eldrup model. \\ One can eliminate the effect of intensity rise in time by sample illumination \\ or by application of high pressure. A decrease in temperature by $1 \mathrm{~K}$ is \\ equivalent to an increase in pressure by about $4 \mathrm{MPa}$.
}

PACS numbers: 36.10.Dr, 61.80.Fe, 71.60.+z, 78.70.Bj

\section{Introduction}

Positron annihilation is a convenient tool to study the structural changes of condensed organic media. A bound system of electron and positron (positronium, $\mathrm{Ps}$ ) is trapped in electron-free regions and the lifetime of its triplet substate (ortho-positronium, o-Ps) depends on the size of free volume. The subject of this study was the dependence of $o$-Ps lifetime and intensity on temperature, pressure and self-irradiation by positron source for a set of odd-numbered $n$-alkanes from $\mathrm{C}_{11} \mathrm{H}_{24}$ (undecane) to $\mathrm{C}_{21} \mathrm{H}_{44}$ (heneicosane). Most interesting for us were the properties of so-called rotator (waxy) phase and the transition rotator-rigid phase, thus, no measurements were done for even-numbered alkanes, as there is no rotator phase in them at carbon chain length below 22 atoms. The rotator phase can be observed in $n$-alkanes with the carbon chains from 11 to 40 atoms long. It is characterized by an orientational disorder; the spatial orientation of long molecule axis is preserved, however the molecules can rotate around that axis. In the rotator phase the non-planar conformers are abundant. This creates relatively large free volumes between the molecules located in the same layer of smectic-like structure of alkane crystal; creation of such free volumes is easily observed in positron lifetime spectra.

${ }^{*}$ corresponding author; e-mail: goworek@tytan.umcs.lublin.pl 


\section{Experimental}

The samples of $n$-alkanes (purity $99 \%$ or better) in liquid phase were placed in the measurement chamber together with positron source in a Kapton envelope (150 kBq of ${ }^{22} \mathrm{Na}$ ) and degassed by freeze-thaw method. The copper chamber was fixed to the upper end of cold finger contacting with liquid nitrogen; just below the chamber a heating coil was placed. The temperature was controlled with the accuracy of $\pm 0.1 \mathrm{~K}$ by Shimaden FP21 regulator with $\mathrm{T}$ type thermocouple. The chamber was evacuated to the air pressure of about $0.5 \mathrm{~Pa}$ to avoid spin conversion in the presence of molecular oxygen dissolved in liquid phase. Two light-emitting diodes (LEDs) were placed inside the chamber. The wavelength of light was $\approx 0.95 \mu \mathrm{m}$, which was enough to make empty the shallow traps accommodating electrons produced by positrons along their ionization tracks.

The measurements with application of high pressure were performed with solid $n$-nonadecane sample only, using a Unipress U-11 gas compressor. To avoid penetration of working gas (argon) to the sample, the pellets of nonadecane were placed in a thin-walled tube closed by two pistons. The tube interior was evacuated through a side opening and then one piston was moved down, below that opening. The volume between the pistons, executing the pressure, was fully filled by the samples.

The positron lifetime spectra were registered using a conventional fast-slow delayed coincidence spectrometer with a time resolution of about $240 \mathrm{ps}$. Each spectrum was collected during $1 \mathrm{~h}\left(3.6 \times 10^{5}\right.$ counts per spectrum). At analyzing the spectra we have assumed the existence of 3 discrete exponential components ascribed to the decay of singlet para-Ps, annihilation of free positrons and the decay of $o$-Ps; the correction for positron absorption in Kapton envelope was also applied. Fitting the components was done by LT programme [1].

\section{Results and discussion}

\subsection{Temperature dependences}

The positron lifetime spectra were measured in the range from $125 \mathrm{~K}$ to about $10 \mathrm{~K}$ above the melting point. As an example of $o$-Ps lifetime $\tau_{3}$ and intensity $I_{3}$ vs. temperature the experimental data for $n$-undecane $\mathrm{C}_{11} \mathrm{H}_{24}$ are shown in Fig. 1. It was noticed by us earlier [2] that the $o$-Ps intensity in alkanes rises with the duration of experiment in a similar way as in polymers [3, 4]. Such a rise is caused by accumulation of electrons, produced along the ionization track of a fast positron. These electrons, next trapped in free volume defects, can be picked by subsequent positrons; the longer is duration of experiment, the larger number of electrons is accessible for positrons in Ps creation process. Electron traps are relatively shallow and one can expel electrons by illumination of samples [5] by visible light or even near infrared. The effect of $I_{3}$ increase due to trapped electrons is well visible in Fig. 1. The sample of $n$-undecane was cooled from liquid state to $125 \mathrm{~K}$ and kept at that temperature during $26 \mathrm{~h}$. The initial $I_{3}$ intensity was $25 \%$, and after that 


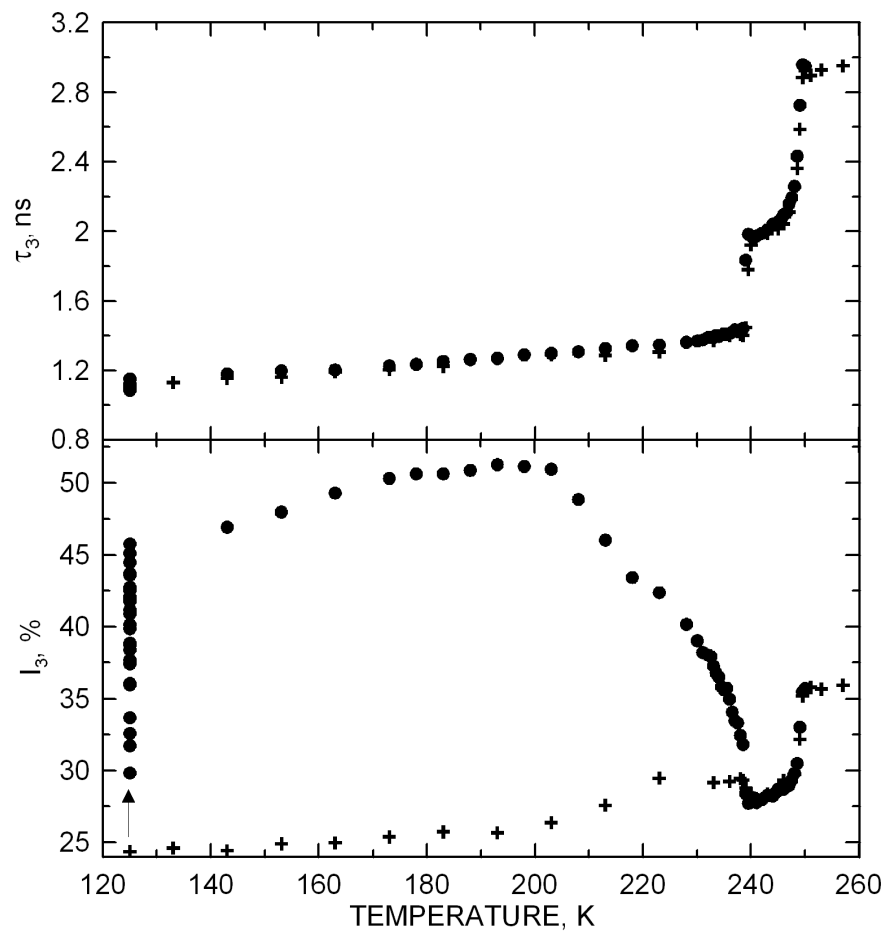

Fig. 1. Temperature dependence of $o$-Ps lifetime $\tau_{3}$ and intensity $I_{3}$ in $n$-undecane after $26 \mathrm{~h}$ storage at $125 \mathrm{~K}$. Dots — without sample illumination, crosses — with illumination by LED (initial storage at $125 \mathrm{~K}$ during $8 \mathrm{~h}$ ).

time it approached the saturation value of about $46 \%$. Then the temperature was increased by steps up to sample melting. A separate run (using a new sample) was performed with the LED switched on, eliminating the $o$-Ps component originating from trapped electrons. One can see that o-Ps component produced by primary processes in the positron blob [6] is almost temperature independent in the whole range of rigid (low temperature) phase. The "trap component" at temperatures below $200 \mathrm{~K}$ is as large as "blob component". Starting from about $200 \mathrm{~K}$ the intensity of "trap component" begins to drop, which is commonly explained as the result of thermal emptying of the traps. From the Arrhenius plot of intensities one can estimate the activation energy of electron escape as $0.4-0.6 \mathrm{eV}[2]$. In the rotator phase and in liquid no effect of trapped electrons is observed. In the whole range of temperatures the lifetime $\tau_{3}$ of $o$-Ps is identical for light on - light off measurements and does not change in time. The $o$-Ps lifetime is determined by the size of free volumes: in the rotator phase - in the vicinity of kink molecules, in the rigid phase - in the gaps between molecular layers [2]. The transition point rigid-rotator in $n$-undecane is easily visible in the lifetime changes, but not in the intensity. 
The "trap component" is the result of initial high energy of positrons from radioactive source and ionization processes accompanying positron slowing down, thus it is not a medium characteristics. For this reason in the measurements presented further below, this component is eliminated by permanent sample illumination with LED. Figure 2 shows the lifetime and intensity of $o$-Ps component for five shortest odd numbered $n$-alkanes in which the rotator phase exists. The trap component in all samples begins to drop at roughly the same temperature $(\approx 200 \mathrm{~K})$ and the slopes of $I_{3}$ vs. $T$ curves above $200 \mathrm{~K}$ are also similar, thus, while in $\mathrm{C}_{11} \mathrm{H}_{24}$ that component extends up to the phase transition point (see Fig. 1), in next alkanes it disappears earlier and is followed by a flat, weakly temperature dependent part. In that range of temperature the effect of $I_{3}$ rise still exists, but amounts several percent only (light bleaching mechanism still works). That can be ascribed to another kind of electron traps, not emptied by temperature increase.

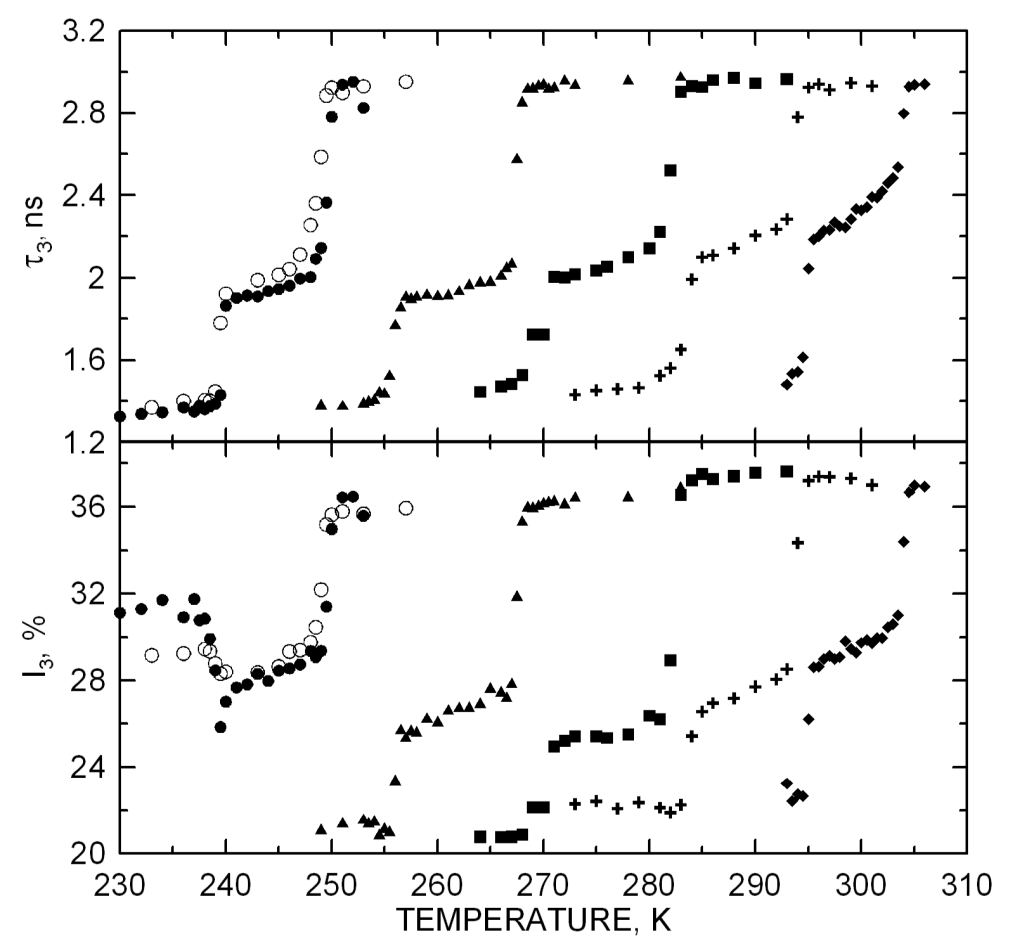

Fig. 2. Temperature dependence of $o$-Ps lifetime $\tau_{3}$ and intensity $I_{3}$ in $n$-alkanes measured with LED switched on. Rising temperature. Dots - undecane (open circles temperature down), triangles - tridecane, squares - pentadecane, crosses — heptadecane, diamonds - nonadecane.

The data shown in Fig. 2 were obtained from the spectra registered at rising temperature. In that figure only the region close to the rotator phase is drawn. 
Two steps on $I_{3}(T)$ curve are visible at the phase transition points: rigid-rotator and rotator-liquid. The lifetime in liquid phase near the melting point is the same for all alkanes under study. According to the "bubble model" [7] that lifetime is determined by surface tension $\gamma$, which increases with the carbon chain length [8], but on the other hand, decreases with temperature. The lifetime was measured near that melting point, which moves to higher temperatures with extending the chain length. Thus, temperature and molecule length act in reverse directions. Besides, the dependence of Ps bubble radius on surface tension is rather weak, like $\gamma^{1 / 4}$.

In the rotator phase, containing a large number of kink-type conformers, the $\tau_{3}$ lifetime at low temperature end of that phase range changes from about $1.9 \mathrm{~ns}$ in $\mathrm{C}_{11} \mathrm{H}_{24}$ to about 2.2 ns in $\mathrm{C}_{19} \mathrm{H}_{38}$. Free volume accommodating Ps atoms in the rotator phase is supposed to have the form of a channel parallel to the molecular chain; its average length is about half of that of molecule, assuming average kink location in the middle of molecule (the position of kink is movable). The relation between molecule length and $o$-Ps lifetime can be described by extended Tao-Eldrup model [9] if one assumes the radius of cylindrical channel $0.24 \mathrm{~nm}$. The cross section of free volume, $0.180 \mathrm{~nm}^{2}$, is found very close to the perpendicular section of the volume occupied by alkane molecule in the crystal structure, $0.185 \mathrm{~nm}^{2}[10]$.

The concentration of kink-shaped molecules in the rotator phase strongly depends on chain length [11] which reflects in the rise of $I_{3}$ step at the rigid-rotator transition. In undecane that step is very small but in heneicosane it reaches $8.5 \%$ (at a very long chain it can be even $12 \%[12]$ ).

\subsection{High pressure effects}

The location of phase transition point is determined by temperature and pressure. In the measurements described above the pressure was zero. In alkanes lowering the temperature is equivalent to increase in pressure approximately by $4 \mathrm{MPa} / \mathrm{K}$ [13]. Figure 3 shows the comparison of temperature and pressure dependence of $o$-Ps intensity and lifetime in $n$-nonadecane $\mathrm{C}_{19} \mathrm{H}_{40}$.

The intensity as a function of rising pressure changes in the same way as that of decreasing temperature when the sample is illuminated. With the light off the intensity $I_{3}$ after temperature induced transition from the rotator to the rigid phase rises rapidly in time and is higher by several percent than with the light on. It means that pressure stops entirely the process of picking electrons from the traps by positron. At the temperature of $302 \mathrm{~K}$, like in Fig. 3, the pressure needed to induce the transition to the rigid phase is $30 \mathrm{MPa}$ or more. The $I_{3}$ rise in time is stopped, moreover, at a very high pressure $I_{3}$ decreases slightly with the time constant of several hours [2]. It seems interesting to see how $I_{3}$ behaves at pressures below $30 \mathrm{MPa}$. In order to check it, the temperature of sample was chosen possibly close to the phase transition point before pressure application. For nonadecane it was $296 \mathrm{~K}$ and for heneicosane - 306.5 K. In spite of quite 


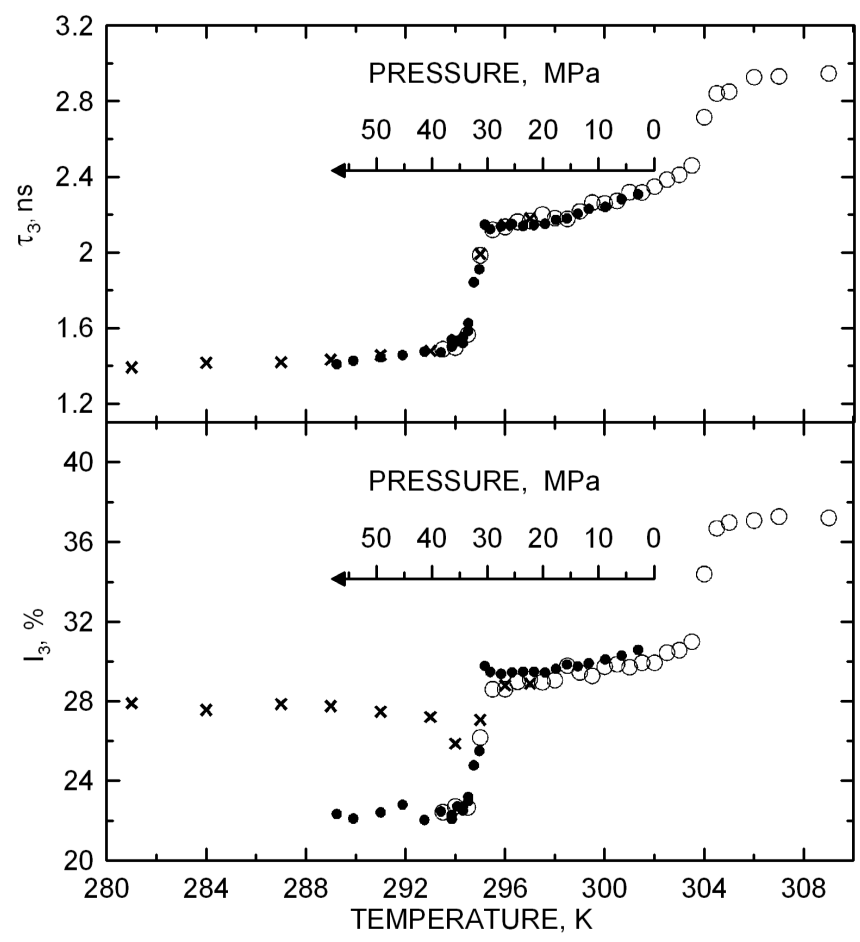

Fig. 3. Ortho-Ps lifetime $\tau_{3}$ and intensity $I_{3}$ in $n$-nonadecane as a function of decreasing temperature. Open circles - with light bleaching, crosses — in darkness. Dots denote measurements at $302 \mathrm{~K}$ as a function of pressure; direction of pressure scale is inverted (shown at the top of picture).

low pressure values ( $8 \mathrm{MPa}$ and $12 \mathrm{MPa}$ at the transition point, respectively) the effect of $I_{3}$ rise with time is eliminated.

\subsection{Long chain alcohols}

Positron lifetime spectra were measured also for $n$-hexadecanol-1, i.e. derivative of $n$-hexadecane with $\mathrm{OH}$ end group. The changes of intensity as a function of temperature are dissimilar to those in $n$-alkanes. In the rotator phase $I_{3}$ is smaller than in liquid and in rigid phases. It follows from increased electric permittivity, reducing the radius of Onsager sphere, and in consequence, the probability to combine $\mathrm{e}^{+}$and $\mathrm{e}^{-}$.

The rotator phase forms at cooling from liquid state only, however in the heating run one observes a narrow dip in $I_{3}(T)$ curve, preceding the melting point. It can be an indication that the traces of rotator phase exist in the range of one $\mathrm{K}$ or less before melting. Contrary to all alkanes the effect of $I_{3}$ change due to irradiation is not observed. Hexadecanol is a polar compound and the potential well in which an excess electron is trapped can be deeper than in the case of alkane; there can be no energy gain at picking an electron by positron and binding the 
particles into positronium. It is interesting that the $o$-Ps intensity is the same for rigid crystal and liquid phases, while in alkanes these intensities are essentially different.

\section{Conclusions}

The relation between $o$-Ps lifetime in $n$-alkanes and free volume size can be described by the version of Tao-Eldrup model for a finite cylinder length. The step of intensity at the transition to the rotator phase is related to the concentration of kink-type conformers. The effect of irradiation by positron source can be eliminated by light bleaching even in near IR range. Also application of high pressure stops the $o$-Ps intensity rise with time; this blocking is effective at the pressures as low as $8 \mathrm{MPa}$. It is advisable to check what is the lower limit of pressure at which the picking of electrons from the traps is still stopped; is it a stepwise dependence or a continuous transition?

\section{References}

[1] J. Kansy, Nucl. Instrum. Methods A 374, 235 (1996).

[2] T. Goworek, R. Zaleski, J. Wawryszczuk, Chem. Phys. 295, 243 (2003).

[3] T. Suzuki, T. Miura, Y. Oki, M. Numajiri, K. Kondo, Y. Ito, J. Phys. IV 3, 283 (1993).

[4] Y. Ito, T. Hirade, E. Hamada, T. Suzuki, Y. Ito, Acta Phys. Pol. A 95, 533 (1999).

[5] T. Hirade, C.L. Wang, F.H.J. Maurer, M. Eldrup, N.J. Pedersen, in: Book of Abstracts of Proc. 35th Annual Meeting on Radioisotopes in the Physical Science and Industries, Tokyo 1998, p. 89.

[6] S.V. Stepanov, V.M. Byakov, J. Chem. Phys. 116, 13 (2002).

[7] Positron and Positronium Chemistry, Eds. D.M. Schrader, Y.C. Jean, Elsevier, Amsterdam 1988.

[8] Handbook of Chemistry and Physics, Ed. D.R. Lide, The Chemical Rubber Co., Cleveland 1988.

[9] T. Goworek, K. Ciesielski, B. Jasińska, J. Wawryszczuk, Chem. Phys. 230, 305 (1998).

[10] J. Doucet, I. Denicolò, A. Craievich, J. Chem. Phys. 75, 1523 (1981).

[11] M. Maroncelli, Song Ping Qi, H.L. Strauss, R.G. Snyder, J. Am. Chem. Soc. 104, 6237 (1982).

[12] T. Goworek, J. Wawryszczuk, R. Zaleski, B. Zgardzińska, to be published in Radiat. Phys. Chem.

[13] H. Forsman, P. Andersson, J. Chem. Phys. 80, 2804 (1984). 